\title{
Functional changes of human quadriceps muscle injured by eccentric exercise
}

F.V. Serrão ${ }^{1}$, B. Foerster ${ }^{2}$,

S. Spada ${ }^{1}$, M.M.B. Morales ${ }^{1}$, V. Monteiro-Pedro ${ }^{1}$,

A. Tannús ${ }^{2}$ and T.F. Salvini ${ }^{1}$

\author{
1Departamento de Fisioterapia, Unidade de Plasticidade Muscular, \\ Universidade Federal de São Carlos, São Carlos, SP, Brasil \\ ${ }^{2}$ Grupo de Ressonância Magnética, Instituto de Física de São Carlos, \\ Universidade de São Paulo, São Carlos, SP, Brasil
}

Correspondence

T.F. Salvini

Departamento de Fisioterapia, UFSCar

13565-905 São Carlos, SP

Brasil

Fax: +55-16-261-2081

E-mail: tania@power.ufscar.br

Presented at the XVII Annual Meeting of the Federação de Sociedades de Biologia Experimental, Salvador, BA, Brazil, August 28-31, 2002.

Research supported by FAPESP (No. 01/12352-1) and CNPq (No. 351197/92-3). S. Spada was the recipient of a fellowship from PIBIC-CNPq.

Received May 8, 2002 Accepted February 11, 2003

\begin{abstract}
The present study evaluated functional changes of quadriceps muscle after injury induced by eccentric exercise. Maximal isometric torque of quadriceps and the surface electromyography (root mean square, RMS, and median frequency, MDF) of the vastus medialis oblique (VMO) and vastus lateralis (VL) muscles were examined before, immediately after and during the first 7 days after injury. Serum creatine kinase (CK) levels and magnetic resonance imaging (MRI) were used to identify muscle injury. The subject was used as her own control and percent refers to pre-injury data. Experiments were carried out with a sedentary 23 -year-old female. Injury was induced by 4 bouts of 15 maximal isokinetic eccentric contractions (angular velocity of $5 \%$; range of motion from $40^{\circ}$ to $110^{\circ}$ of knee flexion). The isometric torque of the quadriceps (knee at $90^{\circ}$ flexion) decreased $52 \%$ immediately after eccentric exercise and recovered on the 5 th day. The highest reduction of RMS occurred on the 2nd day after injury in both VL $(63 \%)$ and VMO $(66 \%)$ and only VL recovered to the pre-injury level on the 7th day. Immediately after injury, the MDF decreased by 5 and $3 \%$ (VMO and VL, respectively) and recovered one day later. Serum CK levels increased by $109 \%$ on the 2 nd day and were still increased by $32 \%$ on the 7 th day. MRI showed large areas of injury especially in the deep region of quadriceps. In conclusion, eccentric exercise decreased the isometric torque and electromyographic signals of quadriceps muscle, which were recovered in one week, despite the muscle regeneration signals.
\end{abstract}

Eccentric exercise has been used as a physiological model to induce muscle injury in humans (1). It is well known that maximal eccentric muscle contractions can produce disruptions in the $\mathrm{Z}$ bands of sarcomeres, especially in muscles of people with sedentary habits (2) or animals (3).

Skeletal muscle injury induced by eccen-
Key words

- Isometric torque

- Surface electromyography

- Quadriceps muscle

- Muscle injury

- Eccentric exercise

- Magnetic resonance imaging tric exercise has been associated with muscle soreness (4), force reduction (5), changes in the electromyographic (EMG) signal (6), and increased serum levels of muscle proteins such as creatine kinase (CK) (7). In addition, magnetic resonance imaging (MRI) of skeletal muscle permits the determination of the location and extent of the injury (8). 
Most of the experiments used to study muscle injury and regeneration have employed invasive methods in animals. Although many mammals have similar processes of skeletal muscle regeneration, it is necessary to improve the evaluation of noninvasive procedures used for the analysis of muscle injury and regeneration in humans. Noninvasive techniques such as surface EMG (9), force (5) and MRI (10) have been used.

In the present study we evaluated the maximal isometric torque of the quadriceps femoral muscle and the electrical activity of the vastus medialis oblique (VMO) and vastus lateralis (VL) daily during the first week of the regeneration process after the muscle injury induced by eccentric exercise. Additionally, in order to detect muscle injury, we determined serum CK levels at three times during the study: before exercise and 2 and 7 days after injury. The MRI of the quadriceps femoral muscle was evaluated before exercise and daily during the 7-day recovery period.

A 23-year-old healthy woman with a sedentary lifestyle gave informed consent to participate in the study, which was approved by the Ethics Committee for Human Studies of the Federal University of São Carlos, São Carlos, SP, Brazil. The participant did not have any orthopedic diseases and had not been previously involved in weight training. The subject was used as her own control and result (\%) refers to pre-injury data.

Eccentric exercise and torque measurements were executed using a Multi-Joint System 2 Isokinetic Dynamometer (Biodex Medical Systems, New York, NY, USA). Muscle injury was induced by eccentric exercise of the right quadriceps muscle. The volunteer was submitted to a total of 4 bouts, each with 15 maximal isokinetic eccentric contractions and a 5-min rest interval between bouts. The contractions were carried out at an angular velocity of $5 \%$ s and the knee flexion ranged from $40^{\circ}$ to $110^{\circ}$.

In order to determine the maximal iso- metric torque of the quadriceps muscle, six independent maximal isometric contractions were performed. Each contraction was maintained for $4 \mathrm{~s}$ with a 2-min rest interval between contractions. The volunteer was positioned in such a way that the hip and knee formed angles of $100^{\circ}$ and $90^{\circ}$, respectively. The mean value and standard deviations of the six measurements were considered for the torque measurements during the study.

The same isometric muscle contractions were used to evaluate both the torque and the surface EMG. In the current experimental setup the instruments for torque and EMG measurements could not be synchronized exactly due to manual initiation of the experiments. However, the small delay of some fractions of a second was negligible compared to the 4-s duration of each contraction. Unlike torque measurement, EMG permits measurements on individual muscle components and was used in the present study to measure VMO and VL.

The electrical activity of the VMO and VL muscles was determined using simple active differential surface electrodes (Lynx Electronics Technologies, São Paulo, SP, Brazil) and a 16-channel signal-conditioning module (1000-V2, Lynx Electronics Technologies). The simple active differential surface electrodes consist of two rectangular parallel bars of $\mathrm{Ag} / \mathrm{AgCl}(1 \mathrm{~cm}$ in length, $0.2 \mathrm{~cm}$ in width and separated by 1 $\mathrm{cm})$. These bars are coupled to a rectangular acrylic resin capsule $2.2 \mathrm{~cm}$ in length, $1.9 \mathrm{~cm}$ in width and $0.6 \mathrm{~cm}$ high. In addition, the electrodes have a common mode rejection ratio with a minimum of $80 \mathrm{~dB}$, an internal gain of 20 times and input impedance higher than $10 \mathrm{G} \Omega$.

The signal-conditioning module (1000V2) was interfaced with a PC computer and had a digital analogue $\mathrm{A} / \mathrm{D}$ converter (CAD 12/32-60K, Lynx Electronics Technologies) with a resolution of 12 bits, an acquisition frequency of $1000 \mathrm{~Hz}$ per channel and an 
Aqdados data acquisition program version 4.6 (Lynx Electronics Technologies). This equipment also has a Butterworth type filter with a bandpass of 10.6 to $509 \mathrm{~Hz}$ and a gain of 50 .

The EMG data were filtered with a bandpass of 10 to $450 \mathrm{~Hz}$ using post-processing procedures based on functions developed to calculate the root mean square (RMS, in $\mu \mathrm{V}$ ) and the median frequency (MDF, in Hz) and introduced in the Matlab 5.0 software. A Hanning window with a $90 \%$ overlap for the processing of Fast Fourier transformation was used to calculate the RMS and the MDF and the data were normalized by the mean.

The preparation of the experiment included shaving and cleaning the volunteer's skin at the positions of the electrodes. Also, palpation of the muscle belly with the subject in the testing position was used to determine electrode placement. The electrodes were fixed with micropore adhesive tape at the midline of the muscle belly with their detection surface perpendicular to the muscle fibers (11). The reference electrode was fixed over the proximal anterior tibia shaft to eliminate possible external interferences. To ensure the reproducibility of the different measurements along the one-week duration of the study, a plastic mold of the quadriceps was elaborated, on which the positions of every electrode were identified.

In a separate experiment but under identical conditions, the contralateral femoral quadriceps of the same volunteer was studied by MRI for visual determination of the effectiveness of eccentric exercise in inducing muscle injury. The MRI of the contralateral quadriceps femoral muscle was evaluated before and during the first 7 days after injury induced by eccentric exercise. The experiments were executed using a 0.5 Tesla NMR scanner developed by the Magnetic Resonance Group of the Physics Institute, São Paulo University, São Carlos, SP, Brazil. Axial images of the thigh were obtained using the inversion-recovery technique with 2000-ms repetition time, 140-ms inversion time and $70-\mathrm{ms}$ echo time. The field of view of the images was $256 \times 256 \mathrm{~mm}$ with a 150 x 250 point acquisition matrix, slice thickness of $15 \mathrm{~mm}$ and 4 averages. Second order flow compensation was used in all three directions.

The two complementary techniques used to investigate muscle injury, torque measurement and EMG were applied once before the eccentric exercise for reference purposes, immediately after the exercise and then on a daily basis to monitor the recovery process over a period of 7 days. In addition to constant monitoring, the serum CK levels were determined at three time points during the study, i.e., immediately before the exercise and on the 2 nd and 7 th day, in order to determine the possible presence of muscle injury. The CK measurements were carried out using the CK-NAC UV kit from Unitest Wiener Laboratory (Rosario, Argentina).
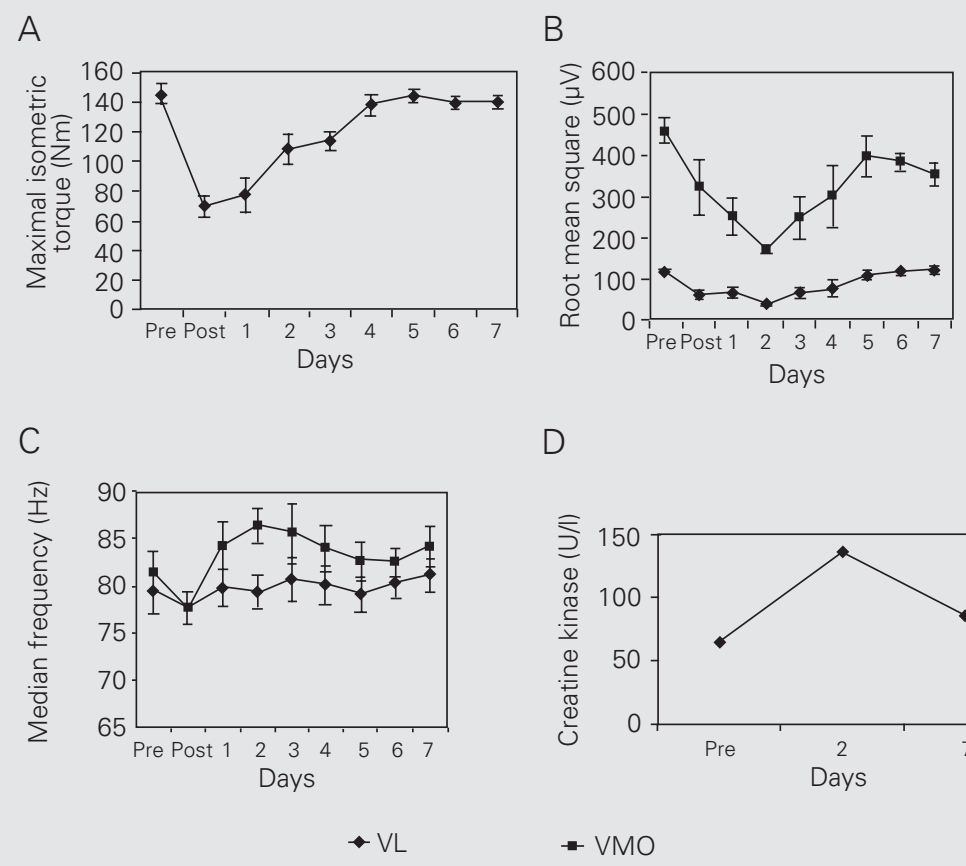

D

Figure 1. Effect of eccentric exercise on maximal isometric torque (A), root mean square (B), median frequency (C), and creatine kinase (D) before (Pre) and immediately after (Post) exercise, and on the 7 days following exercise. Results are reported as means \pm SD for one female subject. $\mathrm{VL}=$ vastus lateralis; $\mathrm{VMO}=$ vastus medialis oblique . 
Figure 1A-C shows the results of torque and EMG measurements. Immediately after the eccentric exercise, there was a decrease in maximal isometric torque, as well as in the RMS and MDF values of both VL and VMO muscles. The maximal isometric torque of the quadriceps muscle decreased by $52 \%$ compared to its pre-exercise level and quickly started to recover, continually improving until reaching its pre-exercise level between the 4th and 5th day (Figure 1A). The MDF values showed a similar behavior (Figure 1C) demonstrating a small initial decrease of 3 and $5 \%$ in VL and VMO, respectively. The RMS measurements also showed a 48 and $30 \%$ decrease of VL and VMO muscles, respectively, but, in contrast to the other experiments, they continued to decrease up to the 2nd day (Figure 1B).

When the recovery period was analyzed (days 1 to 7), a different behavior of maximal isometric torque, RMS and MDF was observed. The maximal isometric torque of the quadriceps gradually recovered to preinjury levels by the 5th day (Figure 1A). Although the largest reduction in RMS for both VMO and VL muscles occurred on the 2nd day after injury (63 and 66\%, respectively), the VL muscle recovered the preinjury values of RMS by the 7th day, while VMO did not recover them until the 7th day (Figure 1B).

The decrease of isometric torque, RMS and MDF, identified in the quadriceps muscle immediately after the eccentric exercise, indicates a failure in the mechanism of muscle fiber contraction, probably associated with muscle fiber fatigue. The difference in RMS between VMO and VL during the regeneration period cannot be easily explained, but one possibility that could be considered is that VMO muscle fibers were injured by the eccentric exercise more than VL fibers. Unfortunately, it was not possible to compare the extent of injury between VMO and VL.

It has been reported that RMS represents the number of active motor units during muscle contraction, and is usually described as a measurement of skeletal muscle activity (12). As the maximal isometric torque is also a measurement of muscle activity, reduction in both isometric torque and RMS, as demonstrated in the present study, may be associated with muscle injury. Previous studies using eccentric exercise to induce injury in the quadriceps muscle also reported a decrease in both muscle force (4) and RMS (13).

Immediately after injury, MDF decreased for both muscles (Figure 1C). A decrease in MDF after eccentric exercise was also observed in other studies $(14,15)$. The decrease in MDF could be attributed to decreased conduction velocity of the active muscle fibers $(16,17)$. Decreased muscle fiber conduction velocity has been associated with proton accumulation (16). Generally, MDF may decrease more if the blood lactate concentration is high, although changes were also observed in the absence of blood lactate (18). Other factors could also be associated with the decrease in MDF, such as impairment of the excitation-contraction coupling (18) and the selective injury of the fast twitch fibers after eccentric exercise (2), which is compensating for an increased activity of the slow twitch fibers trying to maintain force output (14). However, during the recovery period, an increase of both RMS and MDF was observed suggesting that after fatigue associated to muscle fiber injury, more muscle activation was needed to achieve the same relative torque level as pre-exercise level.

The serum level of CK was analyzed only at three times during the study just to confirm the possible presence of muscle injury. Relative to the pre-exercise level, on the 2nd day a pronounced increase of $109 \%$ was observed (Figure 1D), and even by the 7th day CK remained 32\% higher than the first measurement. An increased CK level after eccentric exercise associated with muscle injury has been well documented (7). 
MRI showed large areas of muscle injury, predominantly located in the deep regions of the quadriceps. Observe the hyperintense regions in the images from the 1st to the 7th day after injury (Figure 2) compared to the reference image obtained before exercise. The lesions formed diffuse areas of muscle injury in all regions of the quadriceps muscle, although the deep regions of the muscle appeared to be more affected. Due to the diffuse character of the lesions and the low contrast of the images obtained it was not possible to compare the incidence of injury between VL and VMO, although it
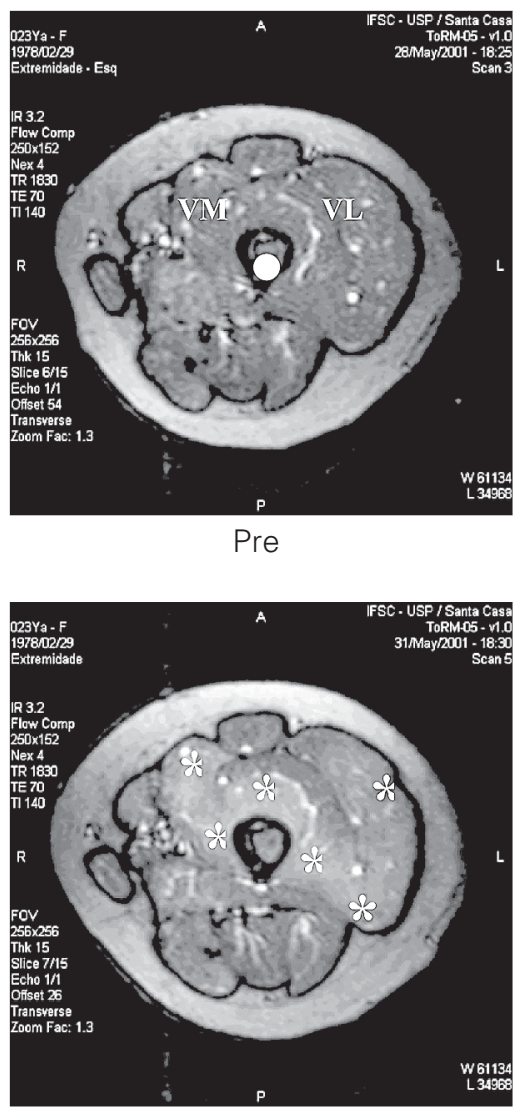

Day 3

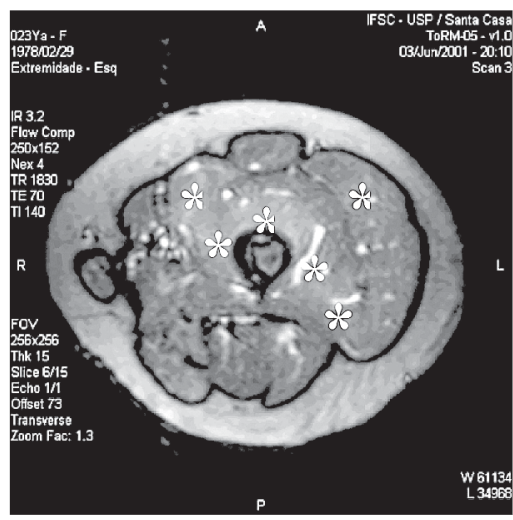

Day 6

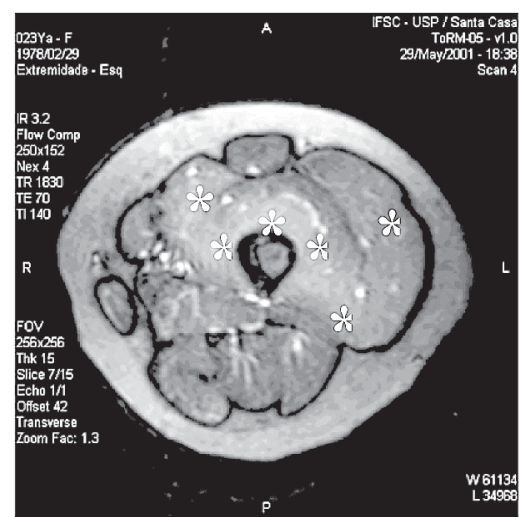

Day 1

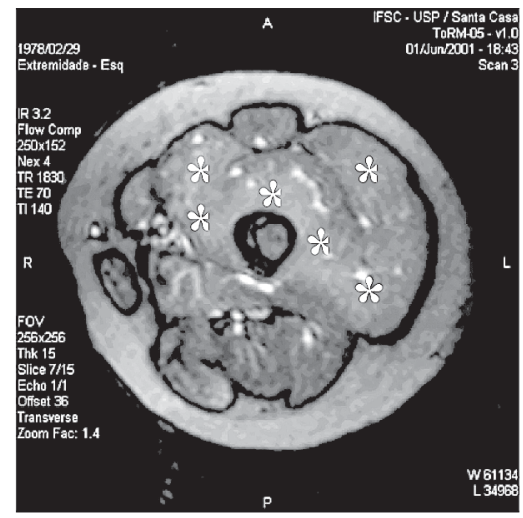

Day 4

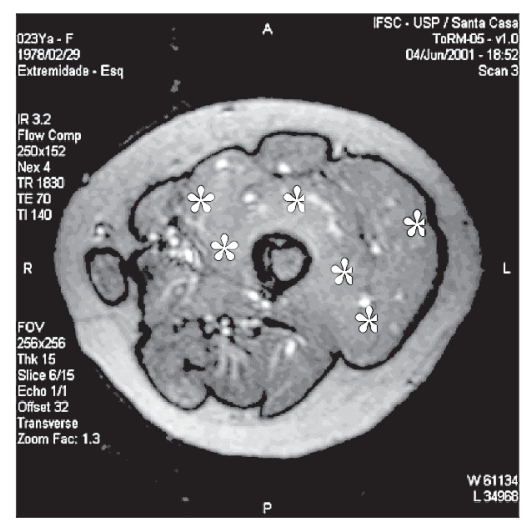

Day 7

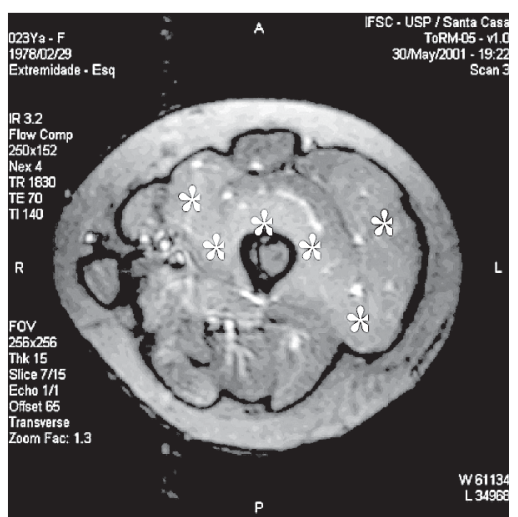

Day 2

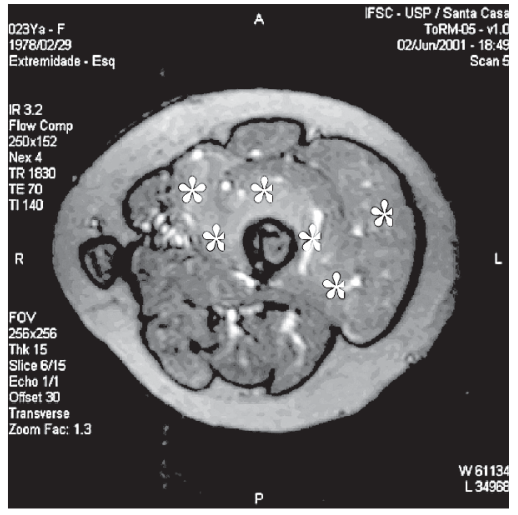

Day 5

Figure 2. Images of the contralateral quadriceps muscle obtained by magnetic resonance imaging (MRI) before eccentric exercise (Pre) and on the 7 days following exercise. Large areas of muscle injury (asterisk) are observed in the quadriceps muscle from the first (day 1 ) to the seventh (day 7) day after eccentric exercise. Femoral bone (Pre, circle), vastus medialis (VM) and vastus lateralis (VL). See text for MRI parameters. 
could be seen that both muscles were affected.

In conclusion, the results of this study demonstrate that bouts of eccentric exercise decrease the isometric torque of the quadri- ceps muscle, as well as the EMG signals of VMO and VL muscles and these changes are recovered within one week, despite the presence of signals of muscle regeneration.

\section{References}

1. Prou E, Guevel A, Benezet P \& Marini JF (1999). Exercise-induced muscle damage: Absence of adaptive effect after a single session of eccentric isokinetic heavy resistance exercise. Journal of Sports Medicine and Physical Fitness, 39: 226-232.

2. Lieber RL \& Fridén J (1999). Mechanisms of muscle injury after eccentric contraction. Journal of Science and Medicine in Sport, 2: 253-265.

3. Clarkson PM (1992). Exercise-induced muscle damage - animal and human models. Medicine and Science in Sports and Exercise, 24: 510-511.

4. Brown S, Day S \& Donnelly A (1999). Indirect evidence of human skeletal muscle damage and collagen breakdown after eccentric muscle actions. Journal of Sports Sciences, 17: 397-402.

5. Rinard J, Clarkson PM, Smith LL \& Grossman M (2000). Response of males and females to high-force eccentric exercise. Journal of Sports Sciences, 18: 229-236.

6. Berry CB, Moritani $T$ \& Tolson H (1990). Electrical activity and soreness in muscles after exercise. American Journal of Physical Medicine and Rehabilitation, 69: 60-66.

7. Thompson D, Nicholas CW \& Williams C (1999). Muscular soreness following prolonged intermittent high-intensity shuttle running. Journal of Sports Sciences, 17: 387-395.

8. Nosaka K \& Clarkson PM (1996). Changes in indicators of inflammation after eccentric exercise of the elbow flexors. Medicine and Science in Sports and Exercise, 28: 953-961.

9. McHugh MP, Connolly DAJ, Eston RG \& Gleim GW (2000). Electromyographic analysis of exercise resulting in symptoms of muscle damage. Journal of Sports Sciences, 18: 163-172.
10. Foley JM, Javaraman RC, Prior BM, Pivarnik JM \& Meyer RA (1999). MR measurements of muscle damage and adaptation after eccentric exercise. Journal of Applied Physiology, 87: 2311-2318.

11. DeLuca CJ (1997). The use of surface electromyography in biomechanics. Journal of Applied Biomechanics, 13: 135-163.

12. Basmajian JV \& De Luca CJ (1985). Muscles Alive: Their Function Revealed by Electromyography. 5th edn. Williams \& Wilkins, Baltimore.

13. Hortobágyi T, Houmard J, Fraser D, Dudek R, Lambert J \& Tracy J (1998). Normal forces and myofibrillar disruption after repeated eccentric exercise. Journal of Applied Physiology, 82: 492-498.

14. Linnamo V, Newton RU, Häkkinen K, Komi PV, Davie A, McGuigan $M$ \& Triplett-McBride T (2000). Neuromuscular responses to explosive and heavy resistance loading. Journal of Electromyography and Kinesiology, 10: 417-424.

15. Linnamo V, Bottas R \& Komi PV (2000). Force and EMG power spectrum during and after eccentric and concentric fatigue. Journal of Electromyography and Kinesiology, 10: 293-300.

16. Lindström L, Magnusson R \& Petersen I (1970). Muscular fatigue and action potential conduction velocity changes studies with frequency analysis of EMG signals. Electromyography, 4: 341-356.

17. Merletti R, Rainoldi A \& Farina D (2001). Surface electromyography for noninvasive characterization of muscle. Exercise and Sport Sciences Reviews, 29: 20-25.

18. Vestergaard-Poulsen $P$, Thomsen C, Sinkjaer T \& Henriksen O (1995). Simultaneous P-NMR spectroscopy and EMG in exercising and recovering human skeletal muscle: a correlation study. Journal of Applied Physiology, 79: 1469-1478. 